\title{
The Management of Acute Onset Complete Heart Block and Atrial Flutter in a Patient with COVID-19
}

\author{
Murtaza Hussain ${ }^{1}$, Dominic Awuah ${ }^{1}$, Smit Deliwala ${ }^{1}$, Mohammed Luay Alkotob²$^{2}$, Elfateh Seedahmed ${ }^{3}$, Ghassan Bachuwa $^{1}$ \\ ${ }^{1}$ Department of Internal Medicine, Michigan State University at Hurley Medical Center, Flint, MI, USA \\ ${ }^{2}$ Department of Internal Medicine, Division of Cardiology, Michigan State University at Hurley Medical Center, Flint, MI, USA \\ ${ }^{3}$ Department of Internal Medicine, Division of Pulmonary Critical Care, Michigan State University at Hurley Medical Center, Flint, MI,USA
}

\section{Doi: 10.12890/2022_003026- European Journal of Case Reports in Internal Medicine - @ E EFIM 2022}

Received: $24 / 10 / 2021$

Accepted: $27 / 01 / 2022$

Published: $23 / 02 / 2022$

How to cite this article: Hussain M, Awuah D, Deliwala S, Alkotob ML, Seedahmed E, Bachuwa G. The management of acute onset complete art block and atrial flutter in a patient with COVID-19. EJCRIM 2022;9: doi:10.12890/2022_003026.

Conflicts of Interests: The authors declare there are no competing interests.

This article is licensed under a Commons Attribution Non-Commercial 4.0 License

\section{ABSTRACT}

Coronavirus disease (COVID-19) has infected millions of people worldwide. Its cardiac presentations include myocarditis, arrhythmias and structural heart changes even in young and healthy individuals. The long-term sequelae of these manifestations are unknown. We describe a unique combination of complete heart block and atrial flutter in the setting of COVID-19. SARS-CoV-2 virulence mechanisms can cause fibrosis in the myocardium resulting in loss of sinus node dominance. The paradoxical finding of atrial flutter and complete heart block is very rare. Prompt cardiac evaluation and electrophysiological testing are important. Cardiac magnetic resonance imaging (cMRI) and endomyocardial biopsies are the gold standard investigations. Anticoagulation should be administered until atrioventricular synchrony is achieved.

\section{LEARNING POINTS}

- Simultaneous atrial flutter and third-degree atrioventricular block (AVB) caused by COVID-19 infection should be treated with a pacemaker according to heart block guidelines.

- During the COVID-19 pandemic, we recommend leadless pacemaker implantation for third-degree AVB as it has a lower risk of infection compared with traditional percutaneous procedures.

- Due to a lack of long-term data, patients with cardiac manifestations from COVID-19 require close follow-up with individualized surveillance schedules.

\section{KEYWORDS}

Complete heart block, atrial flutter, COVID-19

\section{INTRODUCTION}

The spread of coronavirus disease 2019 (COVID-19) after its recognition in Wuhan, China has resulted in a pandemic with huge international implications ${ }^{[1]}$. The majority of cases are asymptomatic or mild, with less than $5 \%$ involving organ failure and decompensation ${ }^{[2]}$. Cardiac manifestations include signs of myocardial injury. A recent meta-analysis reported that cardiac injury was associated with the highest risk of death, ventilator requirement, and in-hospital mortality ${ }^{[3]}$.The majority of patients with conduction disorders experience tachyarrhythmia, with less than $15 \%$ having bradyarrhythmia ${ }^{[4]}$. We describe a very rare case of arrhythmia due to COVID-19. 


\section{CASE DESCRIPTION}

A 32-year-old man presented to the emergency department (ED) with nausea, vomiting, diaphoresis, headaches and intermittent, nonexertional and non-radiating chest pain for 1 week. He did not have a relevant medical history and did not take any home medications, but did use tobacco and marijuana daily. On arrival, his pulse was 30 beats/min, with other vital signs being unremarkable. He was pale, diaphoretic, lethargic and was immediately given atropine and other supportive treatment. An emergent electrocardiogram (ECG) revealed atrial flutter with third-degree atrioventricular block (AVB) with a junctional escape rhythm and QT segment prolongation (Fig. 1). Laboratory workup revealed leucocytosis, elevated brain natriuretic peptide (BNP) and inflammatory markers, as well as negative troponin. The unusual presentation prompted imaging, and computed tomography (CT) from the head to pelvis with contrast that revealed a left subacute cerebellar infarct, ground-glass opacities in the left upper lobe, and a wedge-shaped infarct in the left kidney (Figs. 2-4). As COVID-19 infection was suspected, the patient was swabbed and tested positive. His pulse reached a nadir of 20 beats/min in the ED, with minimal response to atropine. A transthoracic echocardiogram (TTE) demonstrated new-onset cardiomyopathy, an ejection fraction (EF) of 35-40\%, severe biatrial dilatation, pulmonary hypertension, and underlying atrial flutter (Fig. 5).

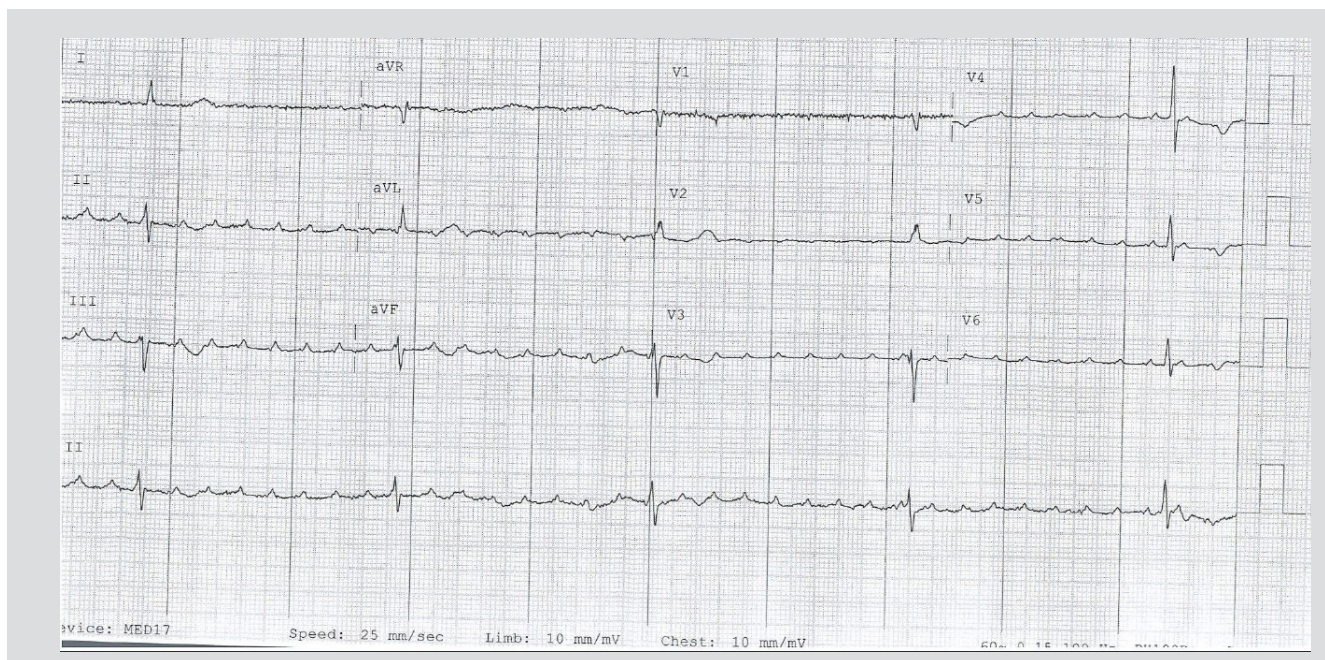

Figure 1. Atrial flutter with complete heart block and escape junction rhythm on electrocardiography (ECG)
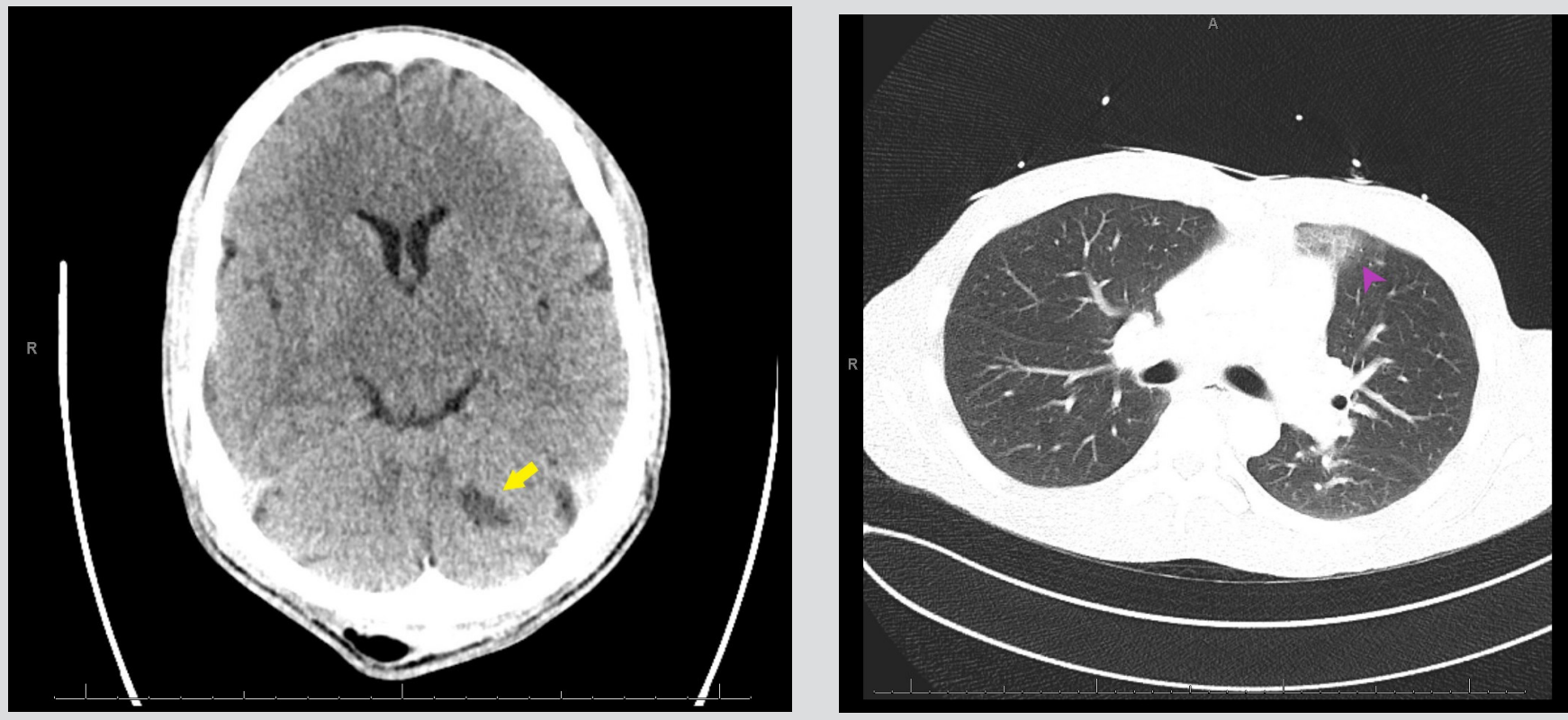

Figure 2. Computed tomography (CT) of the head revealing a subacute left superior

Figure 3. Evolving ground-glass opacity in the left upper lobe on computed tomography cerebellar infarct (arrow)

(CT) of the lung (arrowhead) 


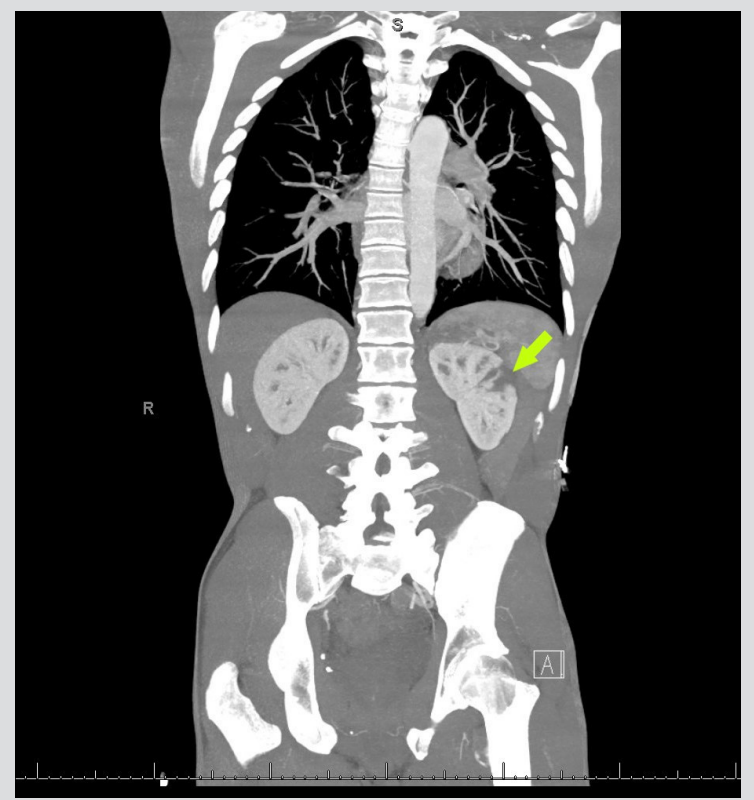

Figure 4. Computed tomography (CT) of the abdomen revealing two low-density areas in the left kidney consistent with renal infarcts (arrow) extremities

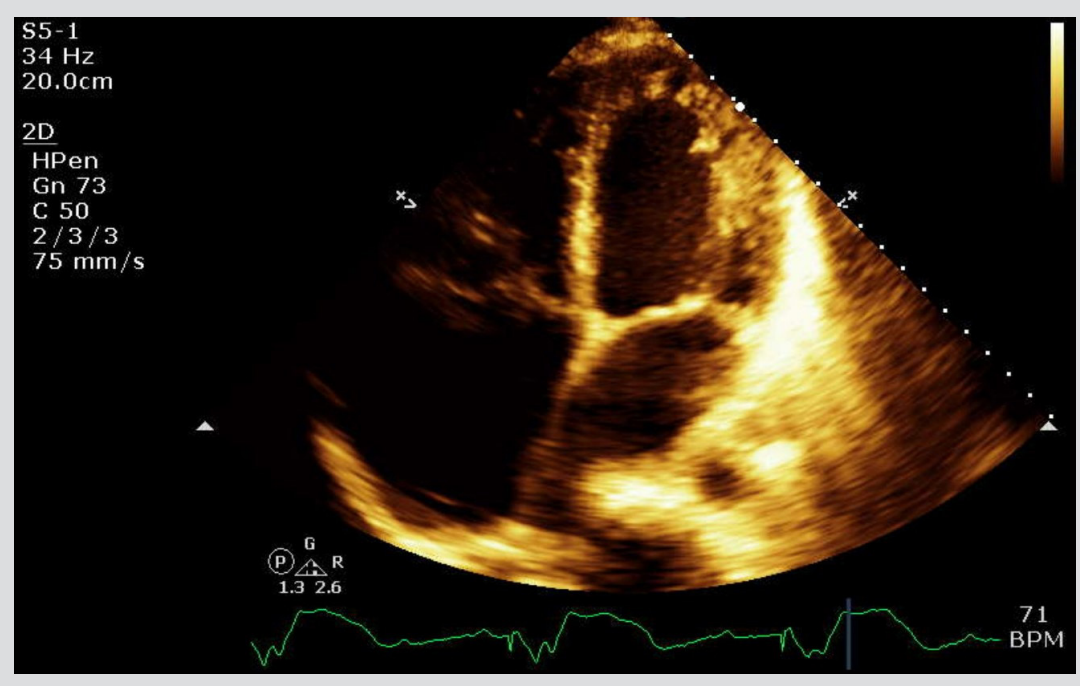

Figure 5. Severely dilated right atrium and moderately dilated left atrium on transthoracic echocardiography (TTE)

The cardiac catheterization procedure room was activated and a temporary pacemaker was eventually placed after initial dislodgement. The patient was admitted to the intensive care unit (ICU), started on a low-dose dopamine drip, and given 6 mg dexamethasone with therapeutic low-molecular-weight heparin (LMWH). CT angiography of the head and neck was negative. Subsequently, a dual-chamber pacemaker was placed without complications. During the electrophysiological procedure, the right atrium was entirely electrically silent, suggesting extensive fibrosis.

The pacemaker was programmed to VVI pacing to avoid atrium tracking.The patient was discharged on a 10-day course of dexamethasone, amoxicillin/clavulanate (800/175 mg) for 7 days, and apixaban $5 \mathrm{mg}$ twice daily. At his 1-month device check follow-up, he denied any additional symptoms and had recovered well without any pacemaker alerts. He was scheduled for cardiac magnetic resonance imaging (MRI) in 6 months and advised to continue anticoagulation.

\section{DISCUSSION}

COVID-19 disease can have numerous clinical sequelae, including cardiovascular manifestations. Our report describes the very uncommon finding of simultaneous atrial flutter and complete heart block in a COVID-19 patient. To our knowledge, this is the first such case described in the literature, although there have been several reports of COVID-19 and heart block, and less frequently of COVID-19 and atrial fibrillation or atrial flutter. The persistence of these dysrhythmias is associated with a poor prognosis, and guidelines from the electrophysiology societies and the European Society of Cardiology recommended that heart failure and arrhythmias caused by myocarditis should be optimally managed with pharmacotherapy, pacing mechanisms, and extracorporeal membrane oxygenation to avoid progression to fulminant myocarditis ${ }^{[5]}$.

Our patient did not display the classic symptoms of COVID-19 pneumonia, but laboratory work-up revealed elevated inflammatory markers, supporting a widespread inflammatory systemic reaction. Moreover, CT imaging demonstrated characteristic ground-glass opacities. This increased our suspicion for COVID-19, with subsequent testing coming back positive. Remdesivir and tocilizumab were not administered due to the patient's stable respiratory status and ongoing comorbidities.

COVID-19 has a spectrum of cardiac manifestations ranging from asymptomatic heart disease to severe infections causing myocarditis and heart failure. Arrhythmias and conduction system disease have also been reported; however, the pathogenesis of arrhythmias in the setting of SARS-CoV-2 is uncertain ${ }^{[6,7]}$. 
In our patient, the atrial enlargement can alternatively be explained by long-standing flutter that led to periodic atrial remodelling, eventually compromising the circuitry during the active phase of the infection and worsening existing scarring and fibrosis, leaving the region 'electrically quiet' (non-conductive myocytes).

Surveillance guidelines for COVID-19-induced myocarditis have not been established. Myocarditis encompasses many entities, and its management is contingent upon the type of sequelae from myocardial damage: heart failure, arrhythmia or myocardial ischaemia. Although our patient presented with concurrent third-degree AVB and atrial flutter, we managed him as having symptomatic thirddegree AVB. Current guidelines indicate that asymptomatic atrial or ventricular premature beats, and non-sustained arrhythmias are not treated. Symptomatic bradycardia with third-degree AVB indicates the need for temporary pacemaker placement (TPM) [8]. If the patient is haemodynamically unstable, then Advanced Cardiovascular Life Support (ACLS) is initiated, and the patient is treated with atropine ${ }^{[9]}$. During the current SARS-CoV-2 pandemic, leadless pacemaker implantation may be a good option as percutaneous intervention decreases the risk of intravascular infection ${ }^{[10]}$. Periodic ECG can be a quick and cost-effective way to delineate early myocardial damage, but results are non-specific. Surveillance of myocarditis should occur every 3 months via a TTE. Cardiac MRI (cMRI) and endomyocardial biopsy (EMB) remain the gold standard investigations for myocarditis confirmation. Anticoagulation should be administered for the atrial flutter until AV synchrony is achieved. We decided against an EMB as it is an invasive procedure and the results would not have acutely changed our management.

\section{REFERENCES}

Bai Y, Yao L, Wei T, Tian F, Jin D-Y, Chen L, et al. Presumed asymptomatic carrier transmission of COVID-19. JAMA 2020;323(14):1406-1407.

Mackenzie JS, Smith DW. COVID-19: a novel zoonotic disease caused by a coronavirus from China: what we know and what we don't. Microbiol Aust 2020;41(1):45-50.

Lakkireddy DR, Chung MK, Gopinathannair R, Patton KK, Gluckman TJ, Turagam M, et al. Guidance for cardiac electrophysiology during the COVID-19 pandemic from the Heart Rhythm Society COVID-19 Task Force; Electrophysiology Section of the American College of Cardiology; and the Electrocardiography and Arrhythmias Committee of the Council on Clinical Cardiology, American Heart Association. Circulation 2020;141(21):e823-e831.

4. Kochi AN, Tagliari AP, Forleo GB, Fassini GM, Tondo C. Cardiac and arrhythmic complications in patients with COVID-19. J Cardiovasc Electrophysiol 2020;31(5):1003-1008.

5. Bao C, Liu X, Zhang H, Li Y, Liu J. Coronavirus disease 2019 (COVID-19) CT findings: a systematic review and meta-analysis. J Am Coll Radiol 2020;17(6):701-709.

6. Newcombe CP, De Souza D, Towers JRH. Atrial flutter with complete heart block. Br Heart J 1960;22(5):691.

7. Peigh G, Leya MV, Baman JR, Cantey EP, Knight BP, Flaherty JD. Novel coronavirus 19 (COVID-19) associated sinus node dysfunction: a case series. Eur Heart J Case Rep 2020;4(FI1):1-6.

8. Figliozzi S, Masci PG, Ahmadi N, Tondi L, Koutli E, Aimo A, et al. Predictors of adverse prognosis in COVID-19: a systematic review and meta-analysis. Eur J Clin Invest 2020;50(10):e13362.

9. Babapoor-Farrokhran S, Gill D, Walker J, Rasekhi RT, Bozorgnia B, Amanullah A. Myocardial injury and COVID-19: possible mechanisms. Life Sci $2020 ; 253: 117723$.

10. Mahdawi TE, Wang H, Haddadin FI, AI-Qaysi D, Wylie JV. Heart block in patients with coronavirus disease 2019: a case series of 3 patients infected with SARS-CoV-2. HeartRhythm Case Rep 2020;6(9):652-656. 